\title{
JURISPRUDENCIA AMBIENTAL EN ANDALUCÍA (PRIMER SEMESTRE 2020)
}

\author{
LORENZO MELLADO RUIZ \\ Catedrático de Derecho Administrativo \\ Universidad de Almería
}


Sumario. 1. Los procesos de regularización no eximen de responsabilidad, pero pueden aminorarla; 2. Responsabilidad patrimonial por daños personales en un sendero de montaña; 3. Naturaleza jurídica (compuesta) de los procedimientos de designación de Zonas de Especial Conservación (ZEC) y obligatoriedad de publicación de las órdenes de desarrollo; 4. Un convenio de colaboración no puede exonerar de su responsabilidad competencial a los entes locales (en materia de saneamiento y depuración de aguas residuales); 5. ¿Puede considerarse "acción popular" la legitimación para el acceso a la justicia en materia de medio ambiente?; 6. ¿Qué se entiende por "cercado cinegético" en relación con la normativa de protección de la flora y fauna?; 7. El instrumento organizativo de la delegación de competencias entre órganos administrativos debe especificar expresamente las funciones delegadas (la delegación del otorgamiento de concesiones demaniales no incluye, así, la de las competencias de resolución de los procedimientos de caducidad de dichas concesiones).

\section{LOS PROCESOS DE REGULARIZACIÓN NO EXIMEN DE RESPONSABILIDAD, PERO PUEDEN AMINORARLA}

STSJA de 16 de mayo de 2019, rec. 609/2016

Resuelve la STSJA (Sala de lo Contencioso-Administrativo, Sección 3ª) núm. 706/2019, de 16 de mayo, rec. 609/2016 (JUR/2019/205067) recurso contra una sanción de multa e indemnización por los daños producidos al dominio público hidráulico a causa del mantenimiento en explotación de ocho captaciones de aguas subterráneas para riego, previo almacenamiento en balsa, sin la preceptiva autorización de la Confederación Hidrográfica del Guadalquivir.

Entre otras alegaciones de defensa, se alega específicamente que estas actividades de riego no cuenta con autorización habilitante, pero sí se encuentran inmersas en procedimientos públicos de regularización. Entiende el TSJA sin embargo que ello no es suficiente: no se ha especificado el trámite exacto o fase concreta en que se encuentran, ni la identificación de los responsables o personas titulares interesadas. Tener solicitada una concesión no implica la legalidad de las actuaciones pretendidamente cubiertas por la misma, puesto que no se ha demostrado la existencia y vigencia de título concesional, sino sólo las expectativas de conseguirlo al tiempo de la denuncia.

Como dice el TSJA, la circunstancia de haberse acogido al proceso de regularización de aprovechamientos no conlleva a considerar despenalizada su 
actuación, pues al tiempo de la incoación del procedimiento sancionador no se había resuelto el proceso concesional con resolución favorable.

Se alegan también determinadas irregularidades probatorias, menos interesantes, pero sí acaba el TSJA con una apreciación importante en relación precisamente con la virtualidad de estos procesos de legalización de actuaciones irregulares, que no pueden subsanar la carencia de título habilitante de actuación pero sí amortiguar en parte la responsabilidad de los imputados.

En parte porque, como se acaba reconociendo, la Administración no prueba suficientemente la calificación y graduación concreta de la sanción, remitiéndose simplemente a los preceptos aplicables. El principio de proporcionalidad sancionadora impone un mínimo de motivación en tal sentido, en orden a la clasificación primero y graduación posterior de las sanciones aplicables en cada caso. Habla así el TSJA de "poca motivación". Pero es que además, se puede tener en cuenta incluso el hecho de encontrarse los recurrentes dentro de dichos procesos de regularización de actuaciones. Termina así el TSJA: "atendidas las circunstancias concurrentes de la regularización de uno de los pozos y hallarse en proceso de regularización con la comunidad de regantes al igual que la balsa de almacenamiento, debe estimarse parcialmente el recurso interpuesto en el sentido de aminorar la multa impuesta, debiendo quedar reducidos los daños al dominio público hidráulico de forma consiguiente".

\section{RESPONSABILIDAD PATRIMONIAL POR DAÑOS PERSONALES EN UN SENDERO DE MONTAÑA}

STSJA de 19 de julio de 2019, rec. $342 / 2016$

Resuelve la STSJA (Sala de lo Contencioso-Administrativo, Sede de Sevilla, Sección 4aㅡ) núm. 838/2019, de 19 de julio, rec. 342/2016 (JUR 2020/13733), recurso contra la desestimación de responsabilidad patrimonial por los daños sufridos por un ciclista en una vía de montaña, sobre la base, más allá de la suficiencia probatoria o no, de la presunción de la existencia de mayores riesgos en ella que en otras vías ciclistas.

El sendero en cuestión, al que podían acceder vehículos autorizados, ciclos y 
vehículos de tracción animal, estaba recién pavimentado, pero existiendo al final una zona de socavones sin señalizar, donde el accidentado introdujo la rueda delantera de su bicicleta, provocándole su caída hacia unas piedras que se encontraban en el margen de dicho camino, contra las que impactó su espalda, causándole gravísimas lesiones.

Se interpone reclamación por responsabilidad patrimonial contra el Ayuntamiento, la Junta de Andalucía y TRAGSA, como responsable de la pavimentación del sendero. Ambas lógicamente se oponen, echándose las culpas unas a otras. La Administración autonómica alega falta de legitimación pasiva, entendiendo que se trata de un camino rural, cuyo mantenimiento corresponde al Ayuntamiento titular del mismo; por su parte, el Ayuntamiento entiende que, en virtud del convenio celebrado con la Consejería correspondiente de la Junta de Andalucía, es ésta la que debe asumir las obligaciones de conservación y mantenimiento.

Zanja esta primera cuestión el TSJA de forma clara: el convenio es el instrumento de colaboración interadministrativa y puesta en común de las competencias propias y compartidas de ambas instituciones, en aras de la mejor gestión del servicio que ambas tienen encomendado, por lo que, lógicamente, y por ello mismo, deben responder de las consecuencias dañosas derivadas del funcionamiento del servicio.

No obstante se niega a continuación la responsabilidad patrimonial por no quedar acreditada la relación de causalidad entre los daños y la acción/omisión de los entes responsables. Como dice el TSJA, "faltan elementos de convicción suficientes como para relacionar fundada y causalmente el daño con el funcionamiento de/ servicio". Hasta aquí normal, pues se alegaba sólo, más allá de la prueba, la falta de señalización del mal estado del firme, lo que había generado una confianza razonable en el ciclista de un firme sin solución de continuidad. Pero con el relato y las pruebas obrantes, el TSJA no estima la relación entre el daño y dicho mal estado del pavimento. Pero añade una argumentación final más discutible: según el TSJA, aunque estuviera permitido entrar con bicicletas legalmente, se trataba de un sendero de montaña que, aunque inicialmente presentara un firme mejorado, ello no puede generar confianza de que se trate de una pista que permita un tránsito distinto al del 
propio de un sendero de montaña. Es decir, que según el TSJA, cualquier sendero de montaña, aunque permita el acceso de bicicletas, no tendría por qué estar acondicionado para ello, al ser sólo un sendero de montaña. Y en él, "la circulación con bicicleta de montaña no deja de ser una actividad peligrosa, cuyos riesgos no pueden ser cubiertos por la comunidad, que se convertiría así en una especie de aseguradora universal de todo lo que pasa alli".

Más allá de la insuficiencia de la prueba, y de las lógicas limitaciones en cuanto a la imputabilidad de los riesgos, bien conocidas a pesar de la caracterización objetiva de nuestro sistema de responsabilidad patrimonial, resulta llamativa no la citada consideración del ciclismo de montaña como actividad peligrosa, que lo puede ser, sino la indiferencia de estimación a tales efectos de las circunstancias y elementos presentes en el caso, donde un sendero de montaña con posibilidad expresa de tránsito de bicicletas de montaña se había pavimentado sólo en una parte, sin señalización ni avisos al respecto.

\section{NATURALEZA JURÍDICA (COMPUESTA) DE LOS PROCEDIMIENTOS DE DESIGNACIÓN DE ZONAS DE ESPECIAL CONSERVACIÓN (ZEC) Y OBLIGATORIEDAd DE PUBLICACIÓN DE LAS ÓRDENES DE DESARROLLO}

\section{STSJA de 25 de septiembre de 2019, rec. 470/2015}

Resuelve la STSJA (Sala de lo Contencioso-Administrativo, Sección 3aㅗ), núm. 1469/2019, de 25 de septiembre, rec. 470/2015 (RJCA/2020/30), la impugnación de diferentes disposiciones generales y órdenes de desarrollo relacionadas con el procedimiento de declaración de determinados espacios y hábitats como Zonas de Especial Conservación (ZEC). Interesa fundamentalmente por acoger y desarrollar la jurisprudencia previa sentada al respecto por el TS, por ejemplo en la Sentencia de 8 de julio de 2014, rec. 1079/2012, relativa a la naturaleza jurídica $-y$ sus eventuales consecuencias impugnatorias- del procedimiento de designación de las ZECs, en el marco de la Directiva 92/43/CEE, de 21 de mayo, relativa a la conservación de los hábitats naturales y de la fauna y flora silvestres (Directiva Hábitats) y el RD 1997/1995, de 7 de diciembre, por el que se establecen medidas para contribuir a garantizar la biodiversidad mediante la conservación de los hábitats naturales y de la fauna y flora silvestres. Esta Directiva tiene por objeto, recuerda el 
TSJA, contribuir a garantizar la biodiversidad mediante la conservación de los hábitats naturales y de la fauna y flora silvestres en el territorio europeo, previendo la creación de una red ecológica coherente de zonas especiales de conservación llamada Natura 2000 (art. 3). Esta Red, compuesta por los lugares que alberguen tipos de hábitats naturales que figuran en el Anexo I y de hábitats de especies que figuran en el Anexo II, debe garantizar el mantenimiento o, en su caso, el restablecimiento, en un estado de conservación favorable, de los tipos de hábitats naturales y de los hábitats de las especies de que se trate en su área de distribución natural.

Nos encontramos, en concreto, con un procedimiento trifásico, compuesto o "por etapas". La primera consiste en la formulación o propuesta de lista, que es la que se recurre en el caso planteado, y que tiene por objeto la delimitación espacial de los lugares que cuenten con hábitats naturales de interés comunitario y hábitats de especies animales y vegetales de interés comunitario. Esta formulación (inicial) corresponde a las Comunidades Autónomas, como simple facilitación formal a la Comisión Europea de la información sobre aquéllos lugares en que, en principio, concurren los requisitos normativos para tal declaración como de importancia comunitaria. En la segunda fase (proyecto de lista), la Comisión Europea procede a la comprobación de las listas de ámbitos LIC remitidas por los Estados miembros, de cuyo examen puede resultar la insuficiencia o suficiencia de los LIC seleccionados por cada uno de los Estados, debiendo completarse en caso de insuficiencia. Esta fase finaliza con la aprobación por la Comisión de la lista definitiva de LIC. Y finalmente, en la tercera etapa, tras la aprobación de los LIC por la Comisión, surge la obligación de los Estados miembros de declarar estos espacios como Zonas Especiales de Conservación, en el menor tiempo posible y, en todo caso, en el plazo máximo de 6 años, acompañando a dicha designación (formal) la fijación necesaria de medidas de conservación que impliquen, en su caso, adecuados planes de gestión, específicos a los lugares o integrados en otros planes de desarrollo, y las apropiadas medidas reglamentarias, administrativas o contractuales, que respondan a las exigencias ecológicas de los tipos de hábitats naturales del Anexo I y de las especies del Anexo II de la Directiva presentes en dichos lugares. 
Por tanto, no es hasta esta última fase cuando los Estados miembros tienen el deber legal de concretar específicamente el régimen de conservación de cada uno de los lugares afectados. En las fases precedentes (objeto de impugnación) no hay, estrictamente hablando, declaración sustantiva formal ni fijación administrativa del régimen de conservación correspondiente, aduciendo la parte recurrente en primer lugar la propia preclusión del plazo legal para declarar la Zona Especial de Conservación, y consecuentemente para aprobar su Plan de Gestión. Habría caducado, pues, la facultad de la Administración para su declaración. EI TSJA no admite tal argumento: como ha señalado la jurisprudencia comunitaria, en el caso de que un Estado miembro no transponga la Directiva dentro del plazo que ella marca, se produciría una violación del derecho comunitario originario, pudiéndose abrir en su caso un procedimiento contra el Estado incumplidor, pero no la caducidad.

Se alega en segundo lugar la indebida modificación de la superficie de la ZEC en relación con la acordada en su día en el LIC.

Recuerda entonces el TSJA la previa STSJA de 12 de enero de 2017, rec. 477/2015 (confirmada por la STS de 28 de enero de 2019), donde se señala que no cabe la impugnación directa por parte de los recurrentes de las resoluciones de las Comunidades Autónomas por las que se aprueban las propuestas de LICs elevadas a la Comisión Europea (ubicadas en la primera fase antes reseñada). Sólo será con ocasión de la impugnación de las disposiciones de desarrollo de los LICs, una vez aprobados formalmente, cuando éstos podrán ser atacados. Concede el TSJA la razón por tanto a la Administración actuante: hay que esperar a la aprobación efectiva de los LICs e impugnar los actos posteriores de aplicación de la misma para tratar de evitar sus efectos, pero no la de los actos de trámite anteriores que, realmente, habrían desaparecido ya de la vida jurídica, por sustitución mediante el acto definitivo. Una vez aprobado y publicado el listado LIC, que integra la Red Natura 2000, son las Administraciones nacionales, las autonómicas en el caso español, las que deben aprobar las Zonas de Especial Protección, que sí pueden ser objeto de impugnación ya, al igual que el resto de disposiciones o actos que incidan en los derechos de los particulares, y en tales procesos será donde deba interesarse en su caso el planteamiento de una cuestión prejudicial 
de validez de la Decisión de la Comisión al TJUE.

Deniega en definitiva el TSJA este segundo motivo de impugnación señalando que la propuesta autonómica sobre lugares de importancia comunitaria pierde su sustantividad desde el momento en que la Comisión Europea aprueba la lista de LICs de la región biogeográfica mediterránea.

Y llegamos así a la última pretensión de los recurrentes, que sí es favorablemente admitida. En este caso se pretende la nulidad de una ZEC específica y de su Plan de Gestión, aprobado por una Orden específica de la Consejería correspondiente. El argumento de los recurrentes es, sustancialmente, la falta de publicación oficial de dicha Orden. Y el TSJA recuerda, primera, la jurisprudencia precedente, según la cual "en la medida que estos planes de gestión prevalecen incluso sobre el contenido de los planes urbanísticos, así como que en su función de desarrollo de los PORN les corresponde la zonificación concreta de los espacios, así como la determinación de las diferentes actividades que puedan desarrollarse en el mismo, entre otros contenidos, parece más lógico considerar a los mismos planes como instrumentos normativos que por imposición del principio de publicidad de las normas deben ser objeto de publicación" (STSJA de 12 de enero de 2017, confirmada por la STS de 28 de enero de 2019). Según el TS, "no puede compartirse la interpretación de las normas efectuada por la Administración recurrente, para mantener que los planes de gestión en cuestión no tienen carácter normativo, planteamiento que tampoco puede acogerse desde la alegación de que, en el caso de los planes controvertidos que se aprueban por la Orden impugnada, sus previsiones [...] no establecen determinaciones de obligado cumplimiento, pues, además de que ha de estarse a la naturaleza que resulta de su configuración legal atendiendo a las previsiones de la normativa comunitaria e interna, que no puede sustituirse por la aplicación concreta que de tales normas se plasme en cada caso, basta ver el contenido de los mismos para apreciar que el Plan establece las prioridades de conservación, así como los objetivos, criterios y medidas para garantizar el mantenimiento o restablecimiento de un grado de conservación de hábitats y poblaciones de interés comunitario, [...], objetivos, medidas y criterios de actuación ejecutivos que, en cuanto se proyectan sobre los correspondientes 
espacios ZEC afectan a quienes tienen titularidades e intereses en el ámbito de los mismos en los términos previstos y objeto de regulación en el Plan".

Dado el carácter normativo del Plan de Gestión aprobado (y de la Orden de aprobación), se impone necesariamente su publicación en el diario oficial correspondiente (hoy, art. 45.3 Ley 39/2015, de 1 de octubre).

Además, en este caso, esta previsión de carácter general se refuerza en la normativa específica que viene a indicar la necesidad de tal forma de publicidad de los planes de gestión (art. 3 de la Ley 42/2007).

Concluye por ello el TSJA señalando que "tal forma de publicación, por su alcance y contenido, no puede equipararse ni entenderse satisfecha en sus efectos por la remisión a la publicidad en otros medios (por ejemplo, una página web, como es el caso), de acceso distinto e indirecto por parte de los destinatarios y afectados por la disposición, a salvo que la propia normativa reguladora del procedimiento de elaboración disponga esa forma específica de publicidad".

Se estima pues parcialmente el recurso, anulando la Orden de 11 de mayo de 2015 (Plan de Gestión) y se desestima el resto de actuaciones impugnadas por los recurrentes.

\section{Un convenio de colaboración no puede exonerar de su responsabilidad competencial a los entes locales (en materia de saneamiento y depuración de aguas residuales)}

STSJA de 17 de octubre de 2019, rec. 749/2017

Resuelve la STSJA (Sala de lo Contencioso-Administrativo, Sección 1aㅡ) núm. 2270/2019, de 17 de octubre, rec. 749/2017 (JUR/2020/13881) recurso interpuesto contra la sanción de multa y la obligación de indemnizar los daños al dominio público hidráulico por parte de un Ayuntamiento por realizar vertidos de aguas residuales procedentes de la red de alcantarillado público del núcleo urbano a un cauce público sin la preceptiva autorización del Organismo de cuenca. Se alega, en su defensa, en una táctica conocida ya, la inexistencia de culpabilidad al carecer el ente local de las infraestructuras necesarias al incumplir la Junta de Andalucía los convenios suscritos en la materia. 
La jurisprudencia, sin embargo, no está acogiendo dichas tesis de exoneración por falta de cumplimiento previo de instrumentos de colaboración (por otro lado necesarios para la evitación de los daños producidos).

Como se vuelve a reiterar en esta Sentencia, aunque sea cierto el incumplimiento por parte de la Junta de Andalucía de las obligaciones dimanantes del convenio de colaboración correspondiente, "ello no exime a la actora del cumplimiento de sus obligaciones ni justifica la renuncia al ejercicio de una de las competencias atribuidas por la Ley". La competencia municipal se conserva y retiene (y por tanto es de obligado desarrollo) sea cual sea la evolución fáctica y normativa, no exonerando la existencia de un convenio de las obligaciones municipales correspondientes.

Aprovecha además el TJSA para recordar que "es responsabilidad de las corporaciones locales el proveer a todas las aglomeraciones urbanas situadas en su municipio del preceptivo sistema de evacuación de aguas residuales, siendo la Corporación local la titular del servicio y de la autorización, y en este sentido debemos recordar que la Directiva 91/271/CEE, del Consejo, de 21 de mayo, sobre el tratamiento de las aguas residuales urbanas, establece dos obligaciones claramente diferenciadas: en primer lugar, las "aglomeraciones urbanas" deberán disponer, según los casos, de sistemas colectores para la recogida y conducción de las aguas residuales y, en segundo lugar, se prevén distintos tratamientos a los que deberán someterse dichas aguas antes de su vertido a las aguas continentales o marítimas, siendo responsabilidad de los municipios el proveer de dichos sistemas de evacuación".

Concluye por ello la Sentencia ratificando que no existe causa de exoneración de culpabilidad por no haberse producido una delegación de la competencia de saneamiento y depuración de las aguas residuales a la Agencia Andaluza del Agua por la suscripción de un convenio de colaboración entre la misma y los distintos consorcios y Ayuntamientos y ser la competencia, en virtud de lo dispuesto en la Ley Reguladora de las Bases de Régimen Local, del municipio o del consorcio que tenga delegadas estas competencias, como era el caso enjuiciado.

\section{5. ¿Puede considerarse "acción popular" la legitimación para el acceso a}




\section{la justicia en materia de medio ambiente?}

\section{STSJA de 11 de noviembre de 2019, rec. 375/2016}

Sin entrar en el fondo del asunto, resuelve la STSJA (Sala de lo ContenciosoAdministrativo, Sede de Granada, Sección 3aa) núm. 2503/2019, de 11 de noviembre, rec. 375/2016 (JUR 2018/77827) una cuestión preliminar sobre la existencia o no de legitimación activa para recurrir en el ámbito específico del acceso a la justicia en materia ambiental. Según la Administración, no existiría tal legitimación activa por parte de los particulares recurrentes para ejercitar la acción popular en asuntos medioambientales recogida en el art. 22 de la Ley 27/2006, de 18 de julio, reguladora de los derechos de acceso a la información, de participación pública y de acceso a la justicia en materia de medio ambiente, incorporando las Directivas 2003/4/CE y 2003/35/CE.

Según el TSJA no es que no tengan tal legitimación, que parece evidente como se comenta a continuación, sino que, realmente, esta normativa no contiene, per se, una verdadera acción popular.

Recuerda en primer lugar el TSJA la consolidada doctrina jurisprudencial previa sobre las reglas, contenido y alcance de la legitimación activa en materia contencioso-administrativa, así como la excepción, en determinadas ocasiones, de la necesidad de acreditar el concreto y específico interés legítimo que vincula al recurrente con la actividad objeto de impugnación, por ejemplo en los casos de reconocimiento legal de la acción popular o acción pública. Se entiende en estos casos que la relevancia de los intereses en juego (legalidad urbanística, protección del medio ambiente, etc.) demanda una protección más vigorosa y eficaz que la que puede proporcionar la acción de los particulares afectados, y por ello cualquier ciudadano que pretenda simplemente que se observe y se cumpla la ley puede actuar.

Pues bien, el caso planteado versa sobre la petición de unos particulares sobre la nulidad de la Resolución de la Delegación Provincial correspondiente de la Consejería de Medio Ambiente de la Junta de Andalucía por la que se otorgaba Autorización Ambiental Unificada a una empresa para la explotación de una cantera de áridos.

Es evidente que, al tratarse de particulares, no se cumplirían las previsiones 
correspondientes de la Ley 27/2006, cuyo art. 22 permite, sí, la libre recurribilidad de actos y omisiones en materia ambiental (sin necesidad de acreditar, pues, interés legítimo alguno) pero por personas jurídicas sin ánimo de lucro, y siempre y cuando reúnan los requisitos establecidos al respecto en el art. 23. Como sentencia el TSJA, los recurrentes no cumplen los requisitos exigidos y la resolución impugnada no incide directamente en sus intereses legítimos.

Pero, añade el TSJA, yendo más allá: la Ley 27/2006, en realidad, no recoge, estrictamente hablando, ninguna acción popular, aunque así se denomine, sino, más restrictivamente, el radio normativo habilitante del régimen sustantivo de los intereses legítimos de los que son titulares las entidades en cuyo favor se reconoce dicha acción. Realmente, de las previsiones anteriores se desprende, no una habilitación ciudadana general para recurrir en defensa de la legalidad (medioambiental), sino el reconocimiento de la legitimación por la conexión de su titular con la relación jurídica de fondo sobre la que versa el pleito, exigiéndosele tanto una dedicación activa en la protección medioambiental como que esa actividad se realice en el ámbito territorial al que extienda su eficacia el acto o la omisión impugnados, lo que supone que éstos afectan al objeto social o institucional que tiene asumidos como propios, incidiendo directamente sobre sus intereses legítimos (F. J. $2^{\circ}$ ).

No habría pues, aquí, acción popular o pública, sino reconocimiento específico de la legitimación activa para recurrir a determinados sujetos para la defensa de los derechos e intereses legítimos colectivos que representan o defienden. Como ha recordado incluso el TS, se trataría, pues, de una legitimación colectiva reconocida a favor de aquellas entidades, aunque en este caso pueda sustentarse en la existencia de intereses difusos, concretados en el que todo ciudadano posee en relación con el medioambiente pero que no alcanza para el reconocimiento pleno del derecho a accionar individualmente frente a la ilegalidad administrativa medioambiental (STS de 16 de mayo de 2007).

\section{6. ¿Qué se entiende por "cercado cinegético" en relación con la normativa de protección de la flora y fauna?}

STSJA de 20 de noviembre de 2019, rec. 353/2019 
Resuelve la STSJA (Sala de lo Contencioso-Administrativo, Sección 3ª , Sede de Sevilla) núm. 2128/2019, rec. 353/2019 (JUR 2020/53075) una cuestión sencilla, pero curiosa, relativa a la relación entre la normativa sobre cotos de caza y la legislación sobre flora y fauna correspondiente.

Se recurre, en concreto, la denegación de solicitud de ampliación de un coto de caza concreto. Según la sentencia de instancia, tras analizar la normativa aplicable contenida en la ley $8 / 2003$, de 28 de octubre, de la flora y fauna silvestres, y valorar el informe de los agentes de medio ambiente, no habría especies de caza mayor que pudieran verse afectadas, salvo jabalíes que utilizan gateras o pasos bajo la malla existente en toda su extensión, por lo que no cabría calificar el cercado en cuestión como cinegético. Por el contrario, los recurrentes entienden que sí se trata de un cercado cinegético debido a su altura superior a 1,40 metros y al hecho de impedir realmente el tránsito de la fauna silvestre.

La cuestión a resolver es, pues, sencillamente, si la naturaleza de la cerca existente constituye o no cercado cinegético en los términos de la Ley 8/2003, de 28 de octubre, de la flora y fauna silvestre.

Y señala el TSJA que, a diferencia de lo sostenido por la Administración, la legislación de flora y fauna aplicada no considera que un cercado de las características que reviste el aquí contemplado constituya óbice para conceder la autorización de ampliación del coto solicitada, y ello por no encontrarnos ante un cercado que pueda calificarse de cinegético con dicha normativa.

La Ley 8/2003 dispone, así, que "los cercados cinegéticos son aquellos destinados a impedir el tránsito de las especies cinegéticas de caza mayor". Estos cercados pueden ser de gestión y de protección. Se entiende por cercado de gestión el que aisle del exterior un determinado aprovechamiento cinegético y por cercado de protección el existente en parte del perímetro de un coto o en su interior destinado a proteger cultivos, ganado, reforestaciones 0 infraestructuras viarias de posibles daños originados por las especies cinegéticas. Si conforme a la prueba practicada no hay especies cinegéticas de caza mayor que puedan verse afectadas por el cercado, salvo jabalíes, el cercado no puede calificarse de cinegético al no impedir el paso de las especies de caza mayor. Y si no merece la consideración de cinegético no 
resulta de aplicación la disposición contenida en el segundo párrafo del art. 65 del Decreto 182/2005, de 26 de julio, por el que se aprueba el Reglamento de Ordenación de la Caza de Andalucía, según la cual está prohibida la construcción de dichos cercados con alambre de espinos o mediante cualquier otro método que produzca quebrando físico a los animales. Ciertamente el cercado tenía dos hilos de alambre de espino en la parte superior, pero el hecho no constituye óbice para la obtención de la autorización solicitada al no encontrarnos ante un cercado cinegético por no impedir el tránsito de las especies de caza mayor, de manera que siendo el cercado de una permeabilidad manifiesta para la fauna mayor se entiende que el mismo aún cuando supere la altura de 1,40 metros y tenga alambre de espino, no puede ser calificado de cinegético.

\section{El instrumento organizativo de la delegación de competencias entre} órganos administrativos debe especificar expresamente las funciones delegadas (la delegación del otorgamiento de concesiones demaniales no incluye, así, la de las competencias de resolución de los procedimientos de caducidad de dichas concesiones)

STSJA de 11 de diciembre de 2019, rec. 841/2016

Versa la STSJA (Sala de lo Contencioso-Administrativo, Sede de Granada, Sección 1ª) núm. 2797/2019, de 11 de diciembre, rec. 841/2016 (JUR 2020/62925) sobre la competencia para declarar la caducidad de una concesión de ocupación del dominio público marítimo-terrestre con un establecimiento concreto de comidas y bebidas.

Se alega por los recurrentes la nulidad absoluta del acto recurrido por haber sido dictado por órgano manifiestamente incompetente.

Antes de resolver, recuerda el TSJA los aspectos fundamentales del régimen competencial de los órganos administrativos, así como la diferenciación entre las figuras de alteración de la competencia de la delegación, como transmisión provisional del ejercicio de las competencias a otros órganos administrativos de la misma Administración, aún cuando no sean jerárquicamente dependientes, o a las entidades de derecho público vinculadas o dependientes de las correspondientes Administraciones Públicas, la descentralización 
(intersubjetiva), como solución organizatoria que pretende una mayor eficacia en el desempeño de las funciones administrativas entre las distintas personas jurídicas concurrentes y la desconcentración (interorgánica), como simple técnica de traslado de competencias de unos órganos superiores a otros inferiores, a fín de aligerar cometidos y aproximar la acción pública a la ciudadanía (calificando por cierto la relación entre estas figuras y su aplicabilidad como de situación de "confusión" tanto en la doctrina como en la jurisprudencia).

Pues bien, a la luz de estos presupuestos teóricos, concluye el TSJA que, teniendo en cuenta el principio de indisponibilidad de la competencia ("irrenunciable", según el art. 12.1 de la Ley 30/1992, y, ahora, el art. 8 de la vigente Ley 40/2015, de 1 de octubre), la transferencia de la Dirección General de Prevención y Calidad Ambiental a favor de la Delegación Territorial provincial correspondiente era una delegación de competencias. $Y$ esta delegación se ceñía al otorgamiento de las concesiones demaniales en el dominio público marítimo-terrestre referidas a establecimientos expendedores de comidas y bebidas, pero no expresamente a la competencia relativa a la resolución de los procedimientos de caducidad de las concesiones previamente otorgadas por esa Dirección General. Si no hubo una delegación genérica y global de competencias en relación a los procedimientos concesionales, no puede aceptarse, señala el TSJA, la existencia de delegaciones implícitas de competencias no referidas o expresamente previstas en dicha delegación. 
